Journal of Computer Science 3 (2): 81-83, 2007

ISSN 1549-3636

(c) 2007 Science Publications

\title{
Design of CHWHG Type Low Frequency Magnetic Fields Generator
}

\author{
${ }^{1}$ Bo Cheng, ${ }^{2}$ Ahmed N. Abdalla, ${ }^{1}$ Xi Huang, ${ }^{1}$ Tong han Lan and ${ }^{1}$ Jiarui Lin \\ ${ }^{1}$ Bioinformation and Control Institute, Huazhong University of Science and Technology \\ Wuhan 430074, China \\ ${ }^{2}$ Department of Electric and Electronics Engineering \\ Huazhong University of Science and Technology, Wuhan, China
}

\begin{abstract}
Highly considering the invariability of magnetic field strength in the Magnetic Field Generators, which used to stimulate rat's nerve cell, surely improve the stimulation performance outcome. A new technique to get an invariable magnetic field strength within Magnetic Field Generator has been proposed, Furthermore it had implemented on microcontroller-based system providing an Alternating Magnetic Field Generator (AMFG) with a high performance. The performance of the system is evaluated using two different methods. The results show that the errors are well and acceptable.
\end{abstract}

Key words: Magnetic field, strength, solenoid winding, frequency

\section{INTRODUCTION}

Polson has reported stimulation of human nerves in-vivo by time varying magnetic fields. And it is realized by driving an intense current wave through a coil placed above the target nervous structures, which will induce a stimulating electric field. Since its first introduction the magnetic nerve stimulation technology evolved rapidly and its applications have been expanded in many clinical areas ${ }^{[1-5]}$. It is expected that functional magnetic stimulation will be greatly utilized in conjunction with functional electric stimulation in some clinical applications in the near future ${ }^{[6-7]}$.

Previously and some how recently, most Electroencephalograph (EEG) recording techniques that use magnetic field to stimulate the rat, have problem concerning the frequency-intensity relation within the magnetic field generators, whereat the intensity reduced while increasing of the frequency which effecting the performance ${ }^{[8-10]}$. The objective of this study was to investigate the Alternating Magnetic Field Generator (AMFG) performance by considering and paying attention particularly on AMFG strength invariability within a wide range of frequencies, and how to realize the results into an Optimized Electronic System.

\section{Hardware implementation}

a. Proposed methodology: To achieve the objectives, $\mathrm{C}$ value should vary while the variation of the frequency $(\omega)$ to give the constant strength $(B)$, so we developed an intelligent controls circuit (based on AT89S8252 microcontroller) to Automate selection of the capacitance value $(\mathrm{C})$.
Array of 13 capacitances is set up, One's value is double of its previous'. Immediately after getting the frequency, the microcontroller will calculate $\mathrm{C}$, then switching the control relays, which had set in series with these 13 capacitances. So we can get a digital capacitance with 13bits precision, as shown in Fig. 1.

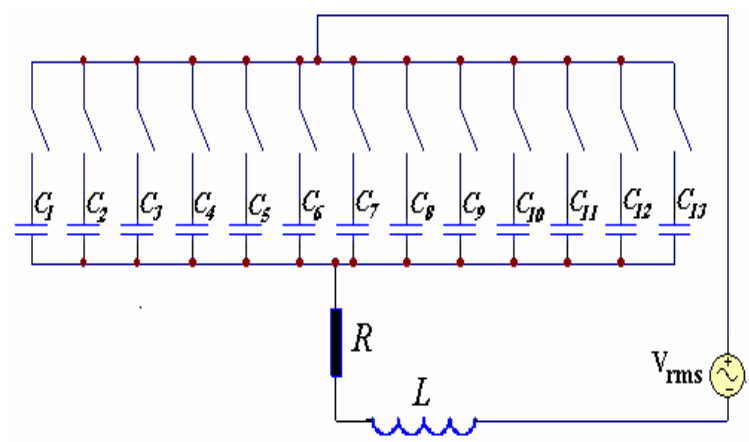

Fig. 1. The capacitances array and its values

b. System design: Figure 2 is a block diagram representation of the designed stimulator. Using the keyboard and the LCD, the microcontroller sends the desired frequency value to the SIN Wave generator chip, which feeds the operational amplifier to amplify the signal. The digitally controlled potentiometer (X9110) is used as feedback resistance to set the operational amplifier (OP27) with a controlled gain and nonlinearity compensation, through a controlled word from the microcontroller. Then the power amplifier maintains the reproduction of the signal at exact amplitude. To overcome the overheating problem a CPU cooler is used. And the microcontroller shut down the power amplifier and the CPU cooler during nosignal periods.

Corresponding Author: $\quad$ Bo Cheng, Bioinformation and Control Institute, Huazhong University of Science and Technology, Wuhan 430074, China 


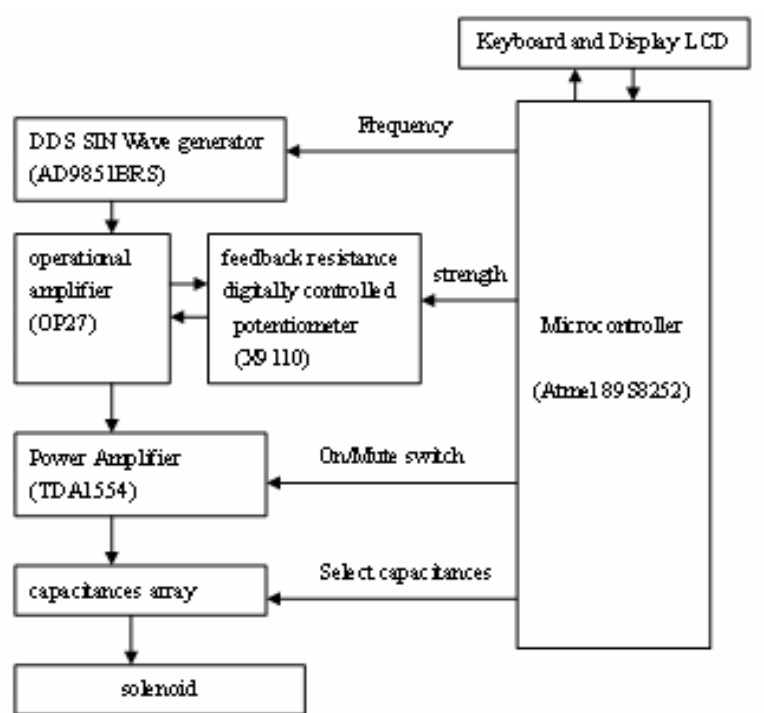

Fig. 2. Block diagram representation of the designed stimulator

c. Algorithm: The algorithm was implemented on a low-power 8-bit microcontroller AT89S8252. This particular microcontroller has been selected based on its features particularly well suited for this project: highperformance CMOS 8 bit microcomputer with $8 \mathrm{~K}$ bytes of Downloadable Flash programmable and erasable read only memory and $2 \mathrm{~K}$ bytes of EEPROM.

Figure 3 shows the description of the microcontroller's program, the software is written in assembler and compiled by Med Win 2.39(a MCU DSK).

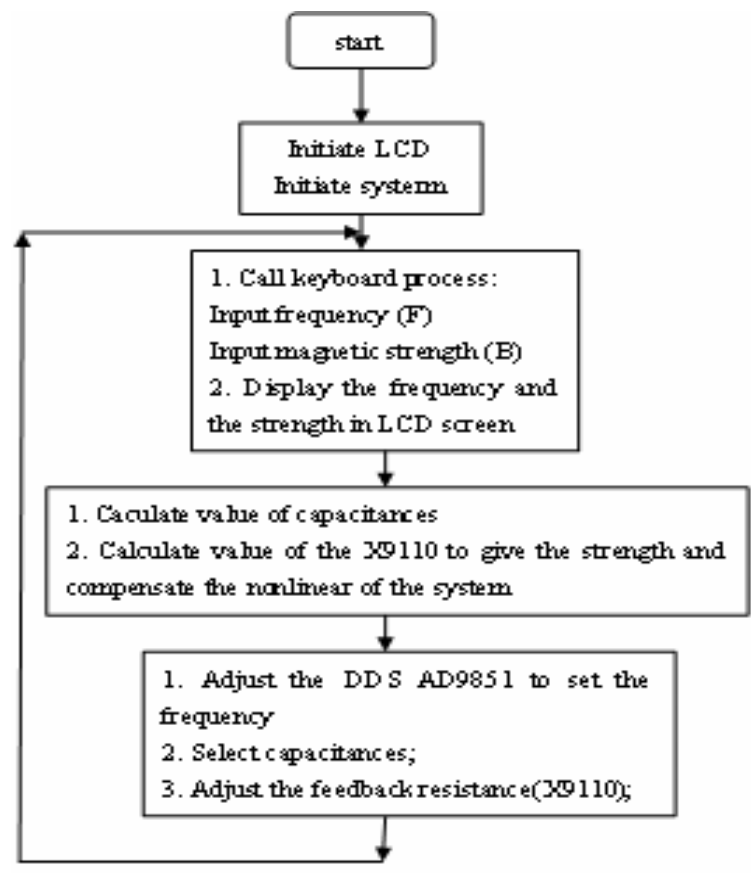

Fig. 3. Microcontroller's program description
Upon power-up, the program initializes the microcontroller; waits user to input the strength and frequency of the magnetic field and display the input two numbers in the LCD. Then the MCU calculates value of capacitances and the control word of the feedback resistance (X9110). Finally the MCU output control signal to the wave generate chip (DDS AD9851) and the digitally controlled potentiometer (X9110).At the same time the MCU control the relays to select the exact capacitances.

\section{RESULTS AND DISCUSSION}

The new technique has been tested and evaluated using two experimental methods, in the first one the magnetic filed strength is measured directly using TJSH-035 Gauss Meter and in the second experiment Faraday's law used to calculate the magnetic field strength as follow.

Figure 4 shows the differences between the conventional method and the new method with digitally

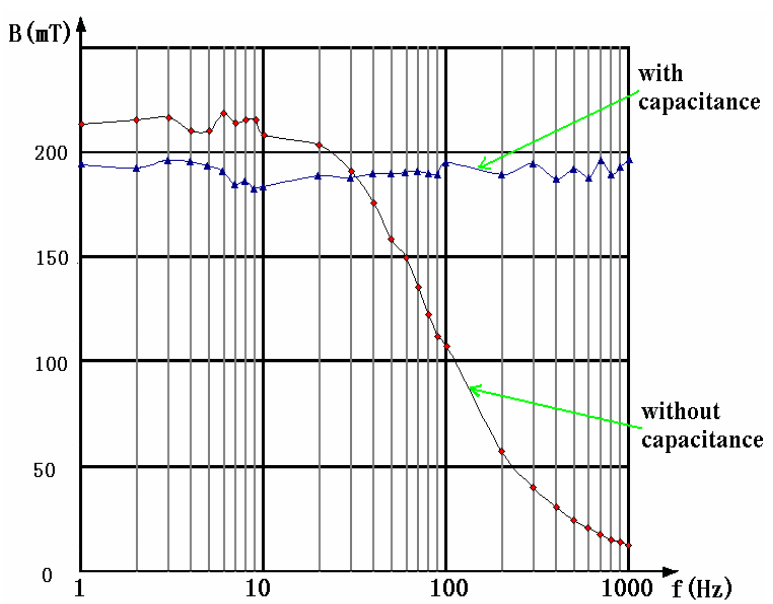

Fig.4. Magnetic field strength versus frequency of the current flowing in the coil

controlled potentiometer, which flatted the strength of the magnetic field generated by the solenoid.

When the frequency $(\mathrm{F})$ varies from $1 \mathrm{~Hz}$ to $1 \mathrm{KHz}$ the magnetic field strength (B) varies from $183.2 \mathrm{mT}$ to 198.1mT. While at the low frequencies (1-10) Hz, the capacitances varies between $(0.0883 \mathrm{~F}$ to $8.83 \mathrm{~F})$, which is too big and difficult to find, we shorted those few capacitances to overcome this difficulty, causing an error (2\%) which is very small and acceptable as shown in Table 1. 


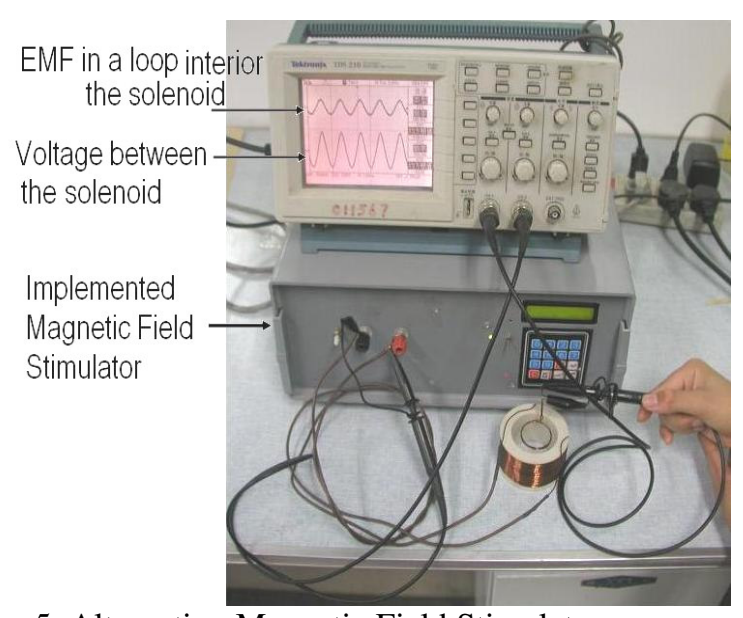

Fig. 5. Alternating Magnetic Field Stimulator

Table 1: System board

\begin{tabular}{lcllll}
\hline $\mathrm{F}$ & $\mathrm{X}=\mathrm{R}+\mathrm{j} \omega \mathrm{L}$ & $\mathrm{C}=1 /(2 \pi \mathrm{f})^{2} \mathrm{~L}$ & $\mathrm{X}=\mathrm{R}+\mathrm{j} \omega \mathrm{L}+1 / \mathrm{j} \omega \mathrm{C}$ & \\
\hline 1 & 1.01 & Short & $8.83 \mathrm{E}+00$ & 1.01 & 1.01 \\
2 & 1.01 & Short & $2.21 \mathrm{E}+00$ & 1.01 & 1.01 \\
4 & 1.01 & Short & $5.52 \mathrm{E}-01$ & 1.01 & 1.01 \\
6 & 1.02 & Short & $2.45 \mathrm{E}-01$ & 1.02 & 1.01 \\
8 & 1.02 & Short & $1.38 \mathrm{E}-01$ & 1.02 & 1.01 \\
10 & 1.03 & Short & $8.83 \mathrm{E}-02$ & 1.03 & 1.01 \\
20 & 1.07 & $2.21 \mathrm{E}-02$ & & 1.01 \\
40 & 1.24 & $5.52 \mathrm{E}-03$ & & 1.01 \\
60 & 1.48 & $2.45 \mathrm{E}-03$ & & 1.01 \\
80 & 1.76 & $1.38 \mathrm{E}-03$ & & 1.01 \\
100 & 2.07 & $8.83 \mathrm{E}-04$ & & 1.01 \\
200 & 3.75 & $2.21 \mathrm{E}-04$ & & 1.01 \\
400 & 7.29 & $5.52 \mathrm{E}-05$ & & 1.01 \\
600 & 10.87 & $2.45 \mathrm{E}-05$ & & 1.01 \\
800 & 14.47 & $1.38 \mathrm{E}-05$ & & 1.01 \\
1000 & 18.07 & $8.83 \mathrm{E}-06$ & & 1.01 \\
\hline
\end{tabular}

The system board as shown in Fig. 5 consists:

* housed within a case of $(330 * 260 * 160 \mathrm{~mm})$;

* more than $100 \mathrm{~W}$ power consumption;

* up to $24 \mathrm{MHz}$ algorithm execution speed.

\section{CONCLUSION}

Large number of applications in the clinical neurological now uses the Magnetic stimulation of the nervous system as a noninvasive technique, which becoming increasingly sophisticated. To improve the performance of the Alternating Magnetic Field Generators, this paper introduced a new technique to get a an invariable magnetic field strength within $10 \mathrm{~Hz}$ to $1 \mathrm{KHz}$ range of frequencies, furthermore this technique is realized by an intelligent electronic design, providing good results. It is to be hoped that the technique will be further developed and consider the pulse magnetic field generator.

\section{ACKNOWLEDGMENTS}

The authors thank Dr. Magdi Amien for his important contributions and valuable discussion. This project supported by National Natural Science Foundation of China and Bioinformation and Control Institute at Huazhong University of Science and Technology (No: 30470413).

\section{REFERENCES}

1. Zhang, J., X. Wang and M. Wang, 1998. Bioeffect of time-varying magnetic fields on sleep. Proc. 20th Intl. IEEE/EMBS Conf., pp: 3300-3302.

2. Polson, M.J.R., A.T. Barker and S. Gardiner, 1982. Stimulation of nerve trunks with time-varying magnetic fields. Med. Biol. Eng. Comput., 20: 243244.

3. Reily, J.P., 1989. Peripheral nerve stimulation by induced electric currents: exposure to time-varying magnetic fields. Med. Biol. Eng. Comput., 27: 101110.

4. Sigang, W., X. Xiaoli and W. Jun, 1998. Design of CXLY-I low-frequency magnetic fields pulse generator. Chin. J. Med. Phys., 15:161-162.

5. Ruohonen, J., P. Ravazzani, F. Grandori and R. Ilmoniemi, 1999. Theory of multichannel magnetic stimulation: Toward functional neuromuscular rehabilitation. IEEE Trans. Biomed. Eng., 46: 646651.

6. Davey, K. and C.M. Epstein, 2000. Magnetic stimulation coil and circuit design. IEEE Trans. Biomed. Eng., 47: 1493-1499.

7. Hsiao, I.N. and V.W.-H. Lin, 2001. Improved coil design for functional agnetic stimulation of expiratory muscles. IEEE Trans. Biomed. Eng., 48: 684-694.

8. Wang, X., Y. Chen, M. Guo and M. Wang, 2005. Design of multi-channel brain magnetic stimulator and ANSYS simulation. IJBEM, 7: 259-262.

9. Xiaoying, N. and F. Changhong, 1999. Curvefitting method in designingmagnetic steel of permanent magnet electrical machine. Small \& Special Elec. Mach., 27: 16-18.

10. xiao-feng, p. and y. ping, 2005. the influence of electromagnetic field irradiated by high- voltage transmission lines on properties of cells. Proc. 2005 IEEE Engg. Med. Biol., 27th Annu. Conf., Shanghai, China. 